\title{
IN VITRO DEGRADATION BEHAVIOURS OF PDO MONOFILAMENT AND ITS INTRAVASCULAR STENTS WITH BRAIDED STRUCTURE
}

\author{
Cong-er Wang ${ }^{1,2}$, Pei-hua Zhang ${ }^{1,2}$ \\ ${ }^{1}$ College of Textiles, Donghua University, Shanghai 201620, China \\ ${ }^{2}$ Key Laboratory of Textile Science \&Technology, Ministry of Education, China \\ E-mail: wangcedaily@163.com, phzh@dhu.edu.cn
}

\begin{abstract}
:
Biodegradable intravascular stent has attracted more and more focus in recent years as an effective solution for angiostenosis. Ideal stents were expected to exhibit sufficient radial force to support the vascular wall, while suitable flexibility for the angioplasty. After vascular remodeling, stents should be degraded into small molecular and be eliminated from human body, causing no potential risk. In this paper, poly-p-dioxanone (PDO) monofilament was braided into net structure with four different braiding density, two of which exhibited sufficient radial force larger than $30 \mathrm{kPa}$, and three of which showed the bending rigidity within 11.7-88.1 $\mathrm{N} \cdot \mathrm{mm}^{2}$. The degradation behaviors of monofilaments and stents have been observed for 16 weeks. The findings obtained indicate that degradation first occurred in morphology region, which induced temporary increase of crystallinity, monofilament bending rigidity and stent mechanical properties. During this period, monofilament tends to be hard and brittle and lost its tensile properties. Then the crystalline region was degraded and stent mechanical properties decreased. All the results reveal that the PDO intravascular stents with braided structure were able to afford at least 10 weeks of sufficient support to the vascular wall.
\end{abstract}

\section{Keywords:}

intravascular stent, braided structure, PDO, compression strength, bending rigidity

\section{Introduction}

Atherosclerosis and other cardiovascular diseases have become a health hazard in recent years. Stent implanted by percutaneous coronary intervention (PCl) is an effective treatment for coronary heart disease caused by vascular stenosis and block [1]. With the method of treatment, stents were implanted to the diseased vessel segment to support the lumen [2]. Drug-eluting metal stents were mostly widely used nowadays. With antistenosis drug coated on the surface, it can be worked to healing. However, as the stent bone were made from metal, its long-term existance in vivo may have potential risk of inflammation, thrombus, and even restenosis [3]. Biodegradable stents were introduced as a 'temporary stent' with the advantage of biocompatibility, nontoxicity and their complete absorption after the healing process; they being the drug carriers had attracted more and more interest [4].

Materials of biodegradable stents can be divided into biodegradable metal, such as Ferro ferrite, magnesium alloy and biodegradable polymer, such as polylactic acid (PLA), polycaprolactone (PCL) and poly-p-dioxanone (PDO) [5] The biodegradable polymers, with better biocompatibility can be modified to control its degradation velocity and get better machinability, were extensively studied [6]. PDO, authorised by FDA for clinical use, has now been applied as a surgical suture, enteric stent and esophageal stent. With the glass transition temperature below $0^{\circ} \mathrm{C}$ and with rich ether bond in molecular chains, PDO monofilament has great flexibility at room temperature which is suitable for stent braiding [7]. Also, it has high crystallinity and high mechanical strength. Animal experiments demonstrated that it has good biocompatibility and that it barely caused inflammation, with a full biodegradable time of 6 months [8]. Marcos [9] and Chu [10] conducted in vitro degradation experiment on PDO suture; however, there has been no long-term ( $>12$ weeks) studies to test the in vitro degeneration properties of PDO intravascular stent.

Stent can be made from sheet, tube, wire and ribbon materials, among which tube and wire were most widely used [11]. The fabrication method mainly depends on raw material choice. Tubes were mostly produced by laser cutting, allowing a slit width of $<20 \mu \mathrm{m}$ to form a pattern. Wires can be formed into stents by coiling, braiding or knitting. Hamm et al. successfully implanted the Strecker stents with a knitting structure into the coronary arteries of 64 patients [12]. Polymers were extruded and drawn into monofilament to get a higher mechanical property, which is suitable for the wire-based fabrication to make stents [13]. The most common wire-based self-expanding stent is the Wall Stent. With its benefits of flexibility and coverage rate, Wall Stent made from PDO has been applied in the treatment of intestinal stenosis [14] and esophageal stricture [15].

Radial force is the foremost property of stent describing the collapse resistance ability to external circumferential pressure, or the strain capacity to external force. It determined whether the stent achieved the correct deployment cling stable to the lumen wall to provide sufficient support, avoiding abrupt closure, maintain blood flowing and obtain the gain of vascular remodeling [16]. There are no general international standard 
testing method and evaluation criterion currently. Rieu et al. and Chen et al. separately tested the stent radial force by water flow stress [17] and air flow stress [18]. These two methods enabled the overall pressure to stents simulating the circumstance in vascular lumen, yet complicated to operate with low accuracy. The platform compression method expresses the radial force indirectly and is easy to handle with close tolerance [19]. As there is no standard value for stent radial force, the result of Rieu [17] was used, which is higher than $30 \mathrm{kPa}$.

Bending rigidity is an important indicator to describe the mechanical property of stents, reflecting the flexibility. Flexibility is a complex of stiffness and pliability. The stiffness made the delivery process easy to handle, while the pliability enabled the deployed stent to follow vessel contour to diseased region and reduce injury to lumen wall [20]. The generally employed testing method was cantilever beam [21], three-point bending [22] and four-point bending [23]. Schmidt [21] used the cantilever beam method to test the bending rigidity of stents, and got the value limitation of self-expanding stents between 11.7 and $88.1 \mathrm{~N} / \mathrm{mm}^{2}$.

This paper communicates the design, preparation and mechanical properties of PDO biodegradable intravascular stents with braided structure, and discusses the in vitro degradation behaviors of PDO monofilament and stents. The productive process of Wall Stent produced from one PDO monofilament by wounding on a brass mould was first described in detail. The long-term in vitro degradation and systematic mechanical tests of PDO stent have not been discussed in other paper before.

\section{Experimental details}

\subsection{Materials}

PDO monofilament (linear density 96tex, breaking strength $133 \mathrm{MPa}$, breaking elongation $48.92 \%$ ) was provided by META BIOMED CO. LTD, Korea. All chemicals were analytically pure and used without further purification.

\subsection{Monofilament behaviours during degradation}

PDO monofilaments were degraded in phosphate-buffered saline (PBS, pH 7.4), preserved in $37^{\circ} \mathrm{C}$ incubators (HH.CP$\mathrm{T}$, Shanghai Sanxin, China). Scanning electronic microscope (SEM, Hitachi, S-4800, Japan) was used to observe the surface morphology during degradation process every 4 weeks. X-ray diffraction (XRD; D/max-2550 PC, Rigaku, Japan) and Differential Scanning Calorimeter (DSC, Pyris-1, Perkin Elmer) were used every 4 weeks to get the crystallinity of monofilaments. Mass loss and tensile properties were tested every 2 weeks after vacuum drying. Single fiber strength tester (LLY06E, Laizhou electron instrument Co., Ltd, China) was used to test tensile properties with the gauge length of $100 \mathrm{~mm}$ and stretch speed of $250 \mathrm{~mm} / \mathrm{min}$. Fabric bending tester (KESFB2, KATO TEKKO CO., LTD) was used to test the bending rigidity of monofilaments. Twenty samples were set paralysed as shown in Figure 1, clipped to the fixture and bending to the opposite direction separately from curvature 0 to 2.5 . Sensor attached to the fixture would got the bending rigidity of PDO monofilament, $\mathrm{N} \cdot \mathrm{mm}^{2}$.

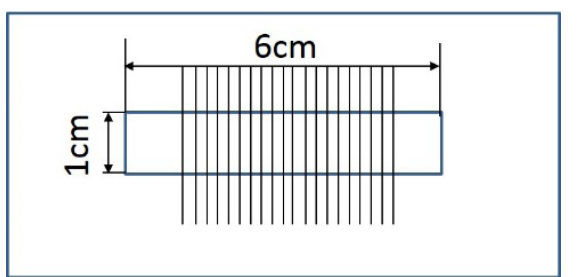

Figure 1. Testing sample of PDO monofilament bending rigidity.

\subsection{Stent preparation}

Figure 2 expressed the process of biodegradable stent implanted and interaction with vascular wall. When fat and cholesterol accumulated to a certain extent, plaque come into being and narrow the vascular lumen. At the same time, endothelial cells were injured, vascular elasticity and pumping capacity receded. Invention treatment was adopted for healing lumen narrow by implanting a compressed stent to the diseased region with a delivery system [Figure 2(a)]. After that, balloon dilated and stent was deployed attaching to the lumen wall, so the vascular was remodeled [Figure2 (b)]. The delivery system then retreated to leave the stent in lumen, which bares the external pressure caused by vascular elastic and movement, as well as the internal pressure of blood flow. Therefore, stents were expected to have sufficient radial force to support the vascular wall during the remodelling period. Otherwise, endothelial cells need to change nutrition and oxygen from blood flow for proliferation, hence the porosity of stent was also necessary to the neointimal growth [Figure 2(c)]. As a temporary stent, after the remodeling process, the biodegradable stents were hydrolysed into small molecules and absorbed by human body or eliminated in vitro. Without existing in vivo permanently, the risk of inflammation and restenosis would be effectively reduce [Figure 2(d)].

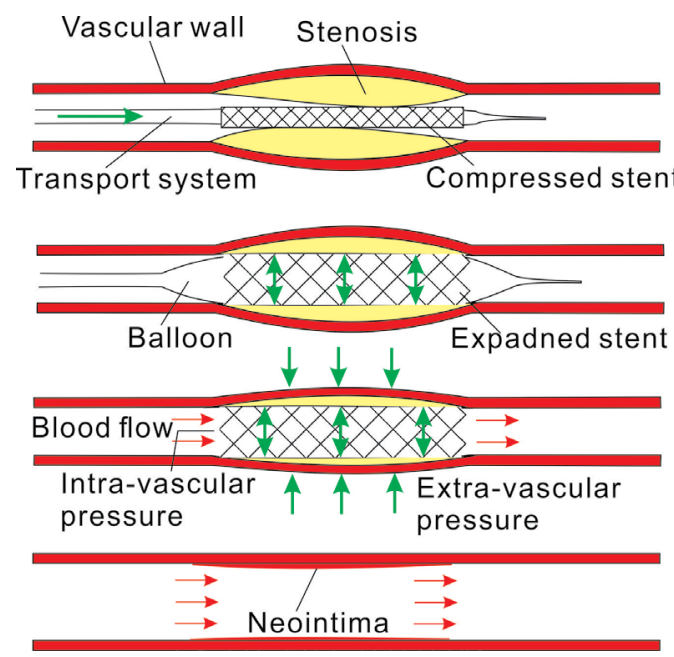

Figure 2. Schematic of biodegradable stent placement process

With the aim of producing stent with a sufficient radial force and bending rigidity as well as porosity, the wire-based stents were prepared by braiding method. Using a PDO monofilament to wound around cylindrical on a brass mould, a length set Wall Stent was formed. Then, the stent was heat setted by electrothermal blowing dryer (Shang Hai Yiheng Co., Ltd, 
China). Demould after cooling. The heat setting parameters are $75^{\circ} \mathrm{C}, 15 \mathrm{~min}$.

Figure 3 showed the wounding path and the expansion draw of stent braiding process. Monofilament was wounded by helix path on a lab-made cylindrical brass mould (diameter $8 \mathrm{~mm}$, length $20 \mathrm{~mm}$ ). On the two head ends of the mould were circular even distributed pins. 6 pin mould was expressed in Figure 3(a). The monofilament started from number 1 pin at the bottom end, helical upward wounded to number 1' pin, fold back and helical down to number 2 pin, and fold back again. Wounded in turn and move one pin step every time in a new cycle, till all pins were done. The monofilament were Interwoven 1/1 up and down to make rhombus net structure stent (stent size: inner diameter $8 \mathrm{~mm}$, length $20 \mathrm{~mm}$ ). By adopting moulds with different number of pins stents with different braiding density could be produced. Use mould with more pins to get stent with higher braiding density, and more compact structure.

As shown in Figure 2(b), the angle $\theta$ between upward and downward helix is called braiding angle.

$$
\theta=\alpha+\beta=\arctan \frac{\pi D}{L}+\arctan \frac{(n+1) \pi D}{n L}
$$

In which, $\alpha$ is the angle between upward helix and the cylindrical mother line, $\beta$ is the angle between downward helix and the cylindrical mother line. $D$ is cylindrical diameter, $L$ is the cylindrical length and $n$ is the number of pins.

Distance between upward helix is $d_{1}$.

$$
d_{1}=\frac{1}{n} \pi D \cos \alpha
$$

While distance between downward helixes is $d_{2}$.

$$
d_{2}=\frac{1}{n} \pi D \cos \beta
$$

Table 1 showed the theoretical value of the braiding angle and helix distance from stents braided with four different mould. It can be seen that, the larger the pin number, the smaller the braiding angle and helix distance, as well as the porosity to make more compact stent. Stent with more compact structure is much more able to resist deformation and has a higher mechanical property. In this paper, four groups stents with different braiding pins were produced, five for each group.

Table 1. The braiding angle and helix distance of stents with different pins

\begin{tabular}{|c|c|c|c|}
\hline $\mathbf{n}$ & $\boldsymbol{\theta}$ & $\mathbf{d 1}$ & $\mathbf{d 2}$ \\
\hline 6 & 107.19 & 2.608 & 2.306 \\
\hline 8 & 106.21 & 1.956 & 1.814 \\
\hline 10 & 105.61 & 1.565 & 1.473 \\
\hline 12 & 105.19 & 1.304 & 1.240 \\
\hline
\end{tabular}

Figure 3 (c) showed the basic structure unit of braided stent, in which

$$
\begin{aligned}
& \mathrm{AB}=\frac{d_{2}}{\sin \gamma}=\frac{d_{2}}{\sin (\pi-\theta)} \\
& \mathrm{BC}=\frac{d_{1}}{\sin \gamma}=\frac{d_{1}}{\sin (\pi-\theta)} \\
& \mathrm{AC}=\sqrt{d_{2}^{2}+C F^{2}}=\sqrt{d_{2}^{2}+\left(B C-\frac{d_{2}}{\tan \gamma}\right)^{2}}
\end{aligned}
$$

When stent compressed, the braiding angel $\theta$ limiting to $0^{\circ}$, height of unit limiting to $A B+B C$. So, theoretically, when stent deployed from the compressed status to $8 \mathrm{~mm}$,

$$
\text { Longitudinal shortage }=\frac{(A B+B C)-A C}{(A B+B C)} \times 100 \%
$$

\subsection{Basic properties test of stent}

Basic properties of stent were described with stent thickness, porosity and longitudinal shortage.

Stent thickness refers to the material thickness of stent wall, which affects not only stent mechanical property but also the cell proliferation rate and inflammation. It can be tested with micrometer.

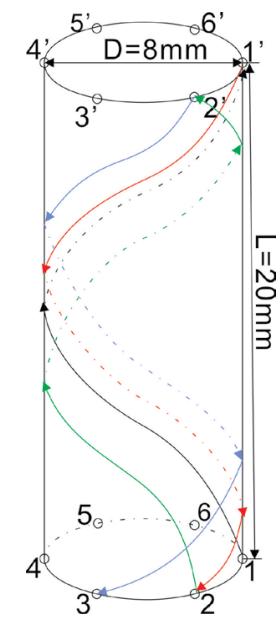

(a)

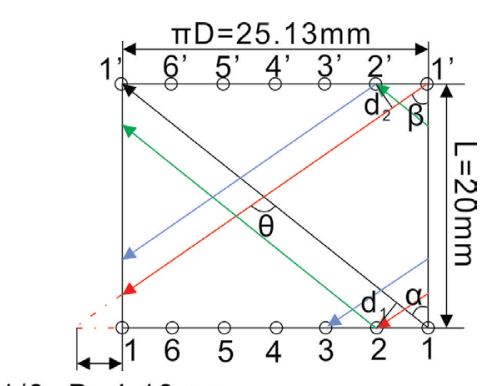

$1 / 6 \pi \mathrm{D}=4.19 \mathrm{~mm}$

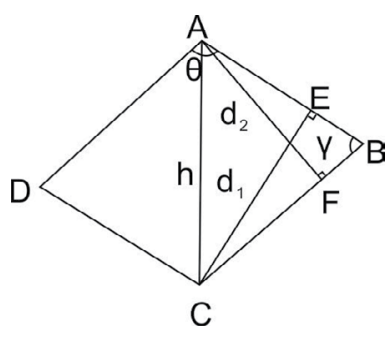

(c)

Figure 3. The schematic diagram of stent design and manufacture of (a) the graphic model, (b) the flat pattern, (c) the basic structural unit. 
Porosity $(K)$ is an important parameter expresses the blank area percentage not been covered by material occupying the whole stent surface area. A certain porosity provided space for cells to proliferate and the environment for gas exchange, nutrition access and metabolite eliminate. Without porous on stent, intima and blood flow were blocked, which would increase the risk of thrombosis and restenosis. However, with an oversize porous, there will be not be enough area for cell proliferation, also decreased stent mechanical property. The porosity could be tested by weight method from Equation (8).

$$
K=\left(1-\frac{m}{\pi \bar{D} L d \rho}\right) \times 100 \%
$$

In which, $\mathrm{m}$ the stent weight, $\mathrm{mg}$; is stent (inner diameter + outer diameter) $/ 2, \mathrm{~mm} ; L$ the stent length, $\mathrm{mm} ; d$ stent thickness, $\mathrm{mm}$; $\rho$ the density of material, $\mathrm{mg} / \mathrm{mm}^{3} \square$

Stent longitudinal shortage is the length shortage of deployed stent compared with the compressed one. Stent with a high longitudinal shortage cannot cover all diseased regions after being implanted into the human body. Micrometer can be used to test the longitudinal shortage, to measure the length at complete compression and deployed to inner diameter $8 \mathrm{~mm}$.

\subsection{Mechanical properties of stents}

Stent mechanical properties were tested by a radial compression tester (YG061, Laizhou electron instrument co., LTD, China) to get the radial force and bending rigidity. Use the platform compression method to test the radial force, as shown in Figure 3 (a). Stent was set on the platform and being compressed by a pressure. Compress the stent to get the deformation of $50 \%$ of its outer diameter, with the pressure rate $20 \mathrm{~mm} / \mathrm{min}$, and pressure diameter $20 \mathrm{~mm}$. The radial force $P$ applied on stent at the largest deformation can be calculated by Equation 9 .

$$
\mathrm{P}=\frac{F_{1}}{S \times(1-K)}
$$

In which, $F_{1}$ is the compression strength at the largest deformation, $S$ is the surface area of stent, while $K$ is the porosity.

Stent bending rigidity can be measured by cantilever beam method, shown in Figure 3 (b). Stent was fixed at one end and compressed by pressure at the other end. The cantilever beam length counts from the fixed point to pressure center line. As known from cantilever beam theory, the stent bending rigidity can be calculated by Equation 10 .

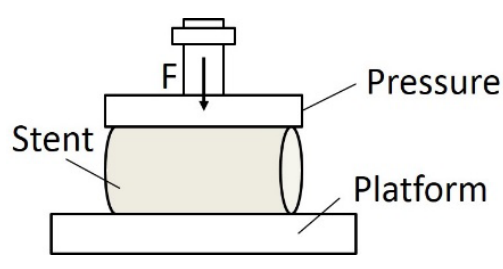

(a)

$$
E I=\frac{F_{2} \times B^{3}}{3 \delta}
$$

In which, $F_{2}$ is the bending strength when stent bending to the largest deformation, $B$ is the cantilever beam length, $\delta$ is the bending deformation. Stent bending rigidity can be line regression calculated from the $F-\delta$ graph. Cantilever beam length is $12 \mathrm{~mm}$, largest bending deformation is $2 \mathrm{~mm}$, pressure rate is $20 \mathrm{~mm} / \mathrm{min}$, and the pressure diameter is $5 \mathrm{~mm}$.

During testing process, soft rubber film (thickness $0.05 \mathrm{~mm}$ ) was used to coat the stent, in order to get more equally force and simulated the pressure status in vascular lumen. Rubber film has no effect to testing results.

\subsection{Stent behaviors during degradation}

Use phosphate-buffered saline (PBS, pH 7.4) for the in vitro degeneration of PDO stents, same process with the monofilament. Measure the stent weight, radial force and bending rigidity every 2 weeks.

\subsection{Statistical analysis}

Statistical analysis was performed using Origin (Origin lab, USA). Data results were averaged and expressed as mean \pm standard deviation (SD). Statistical differences were determined by one-way ANOVA. The statistical significance was assessed by $F$ test, significance level was $p<0.05$.

\section{Results and discussion}

\subsection{Monofilament behaviors during degradation}

Figure 5 were SEM micrographs of PDO monofilament during degradation process. Figure 4 (a) showed the undegraded morphology, with micropit injury got from spinning. With the degradation move on, the longitudinal groove were becoming obvious and more micropits turned up. At the 12th week, it can be seen apparently in Figure 4 (b), there were longitudinal splitting on the surface. When it went to the 16th week, shown in Figure 4 (c), sheet-like peeling occurred on the surface.

Figure 6 expressed the weight retention of PDO monofilament during degradation. During the first 8 weeks, microweight decrease occurred and only $3 \%$ loss at the 10 th week. At this period, hydrolysis broke the ester bond of molecular chains and replaced it by stable ether bond. There were rarely small molecular pieces come into being to cause obvious weight

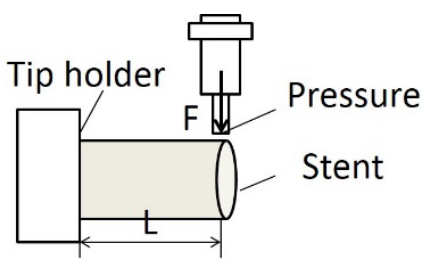

(b)

Figure 4. Schematic representation for PDO stent test of (a) compression strength test, (b) bending rigidity test. 
loss. At 10th week, weight dropped obviously. At this time, during the hydrolysis, more small molecular pieces separated from monofilament into solution. At the 14th week, there were visible pieces getting off the monofilament and the weight retention at 16 th week was $80.42 \%$. This result can also be demonstrated by SEM graphs, which the splitting and sheetlike peeling after 12th week caused a dramatic decrease of monofilament weight.
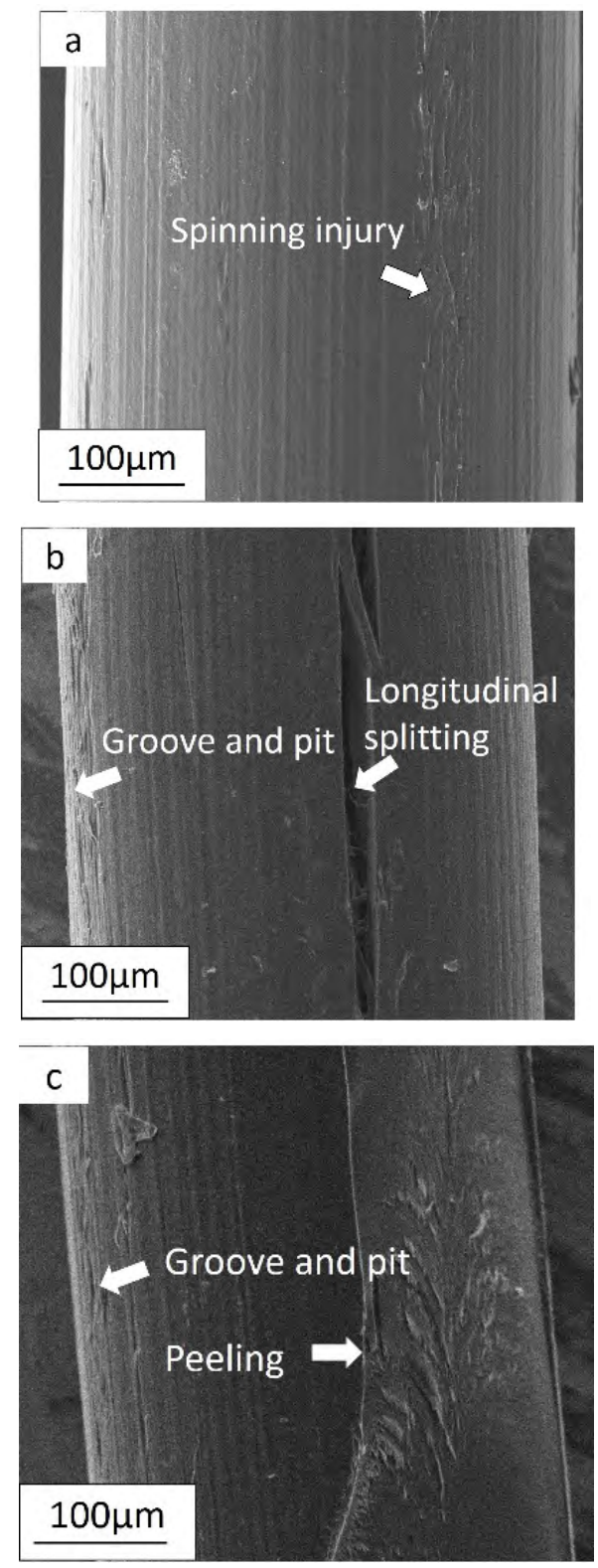

Figure 5. SEM micrographs of PDO monofilament surface during degradation of (a) 0week, (b) 12week, (c) 16week.

XRD patterns of PDO monofilament during degradation period was shown in Figure 7. Undegraded sample showed two peaks at $21.62^{\circ}$ and $23.47^{\circ}$ separately, which indicated that the material had crystalline region. With degradation went on, peaks moved rightward slightly, separately at $22.13^{\circ}$ and $23.98^{\circ}$ at 8 th week. Then the peaks moved leftward to $21.87^{\circ}$ and $23.67^{\circ}$ at 16 th week. The height and area of these two peaks also increased first then declined during degradation process. The crystallinity of undegraded PDO was $70.36 \%$, and increased to $81.31 \%$ at 8 th week, while decreased to $71.61 \%$ at 12 th week, then
$67.88 \%$ at 16 th week. That is because during the degradation period, hydrone first -broke the amorphous region connection the molecular chains there and degraded this region. That induced the increased capacity of crystalline region and cause increased crystallinity. However, the degradation moved on, hydrone kept getting into the compact crystalline region to hydrolyse this region and caused the drop of crystallinity.

DSC was tested every 4 weeks, as shown in Figure 8. The tested melting point $\left(T_{\mathrm{m}}\right)$ and enthalpy $\left(\Delta H_{\mathrm{m}}\right)$ were listed in Table 2. At the first 8 weeks, there were two melting peaks at the curve, as PDO is a semicrystalline material and tend to form its own crystalline region. Then, with the degradation going on long molecular chains were broke to shorter ones, molecular distribution got closer and peaks drawn upon each other and finally join one peak at the 12th week. Though DSC testing can't get the crystallinity directly, it can also be demonstrated that the changes of crystallinity in accordance to the XRD results, as it is in direct proportion with melting enthalpy $\left(\Delta H_{\mathrm{m}}\right)$, which also first grow and then drops. As errors existed among different testing method, there was a certain difference with the result of XRD.

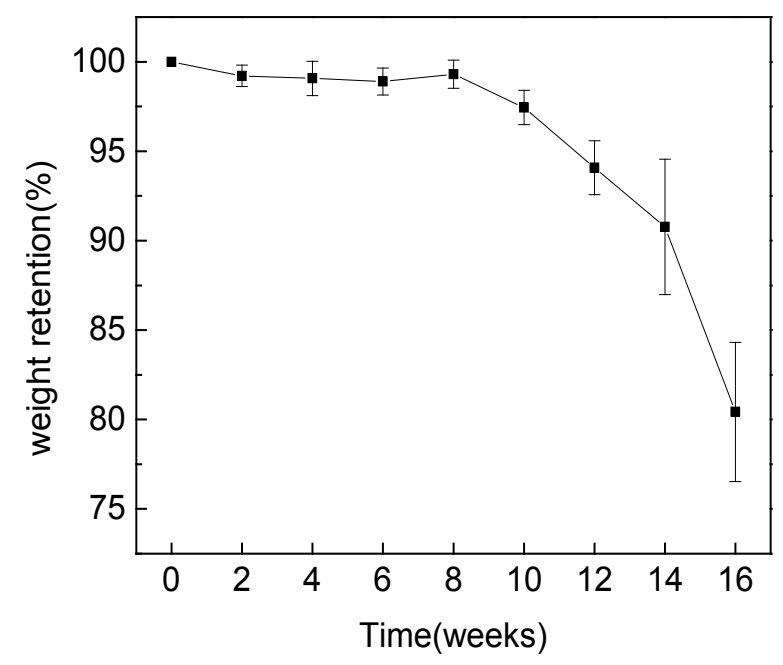

Figure 6. Weight retention of PDO monofilament during degradation. Data were mean $\pm S D, n=5$.

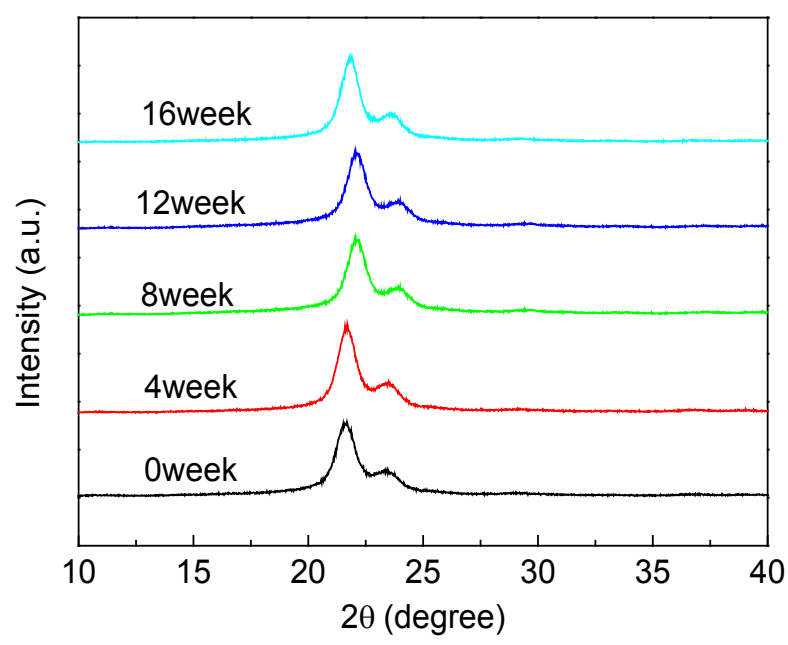

Figure 7. XRD patterns of PDO monofilament during degradation. 


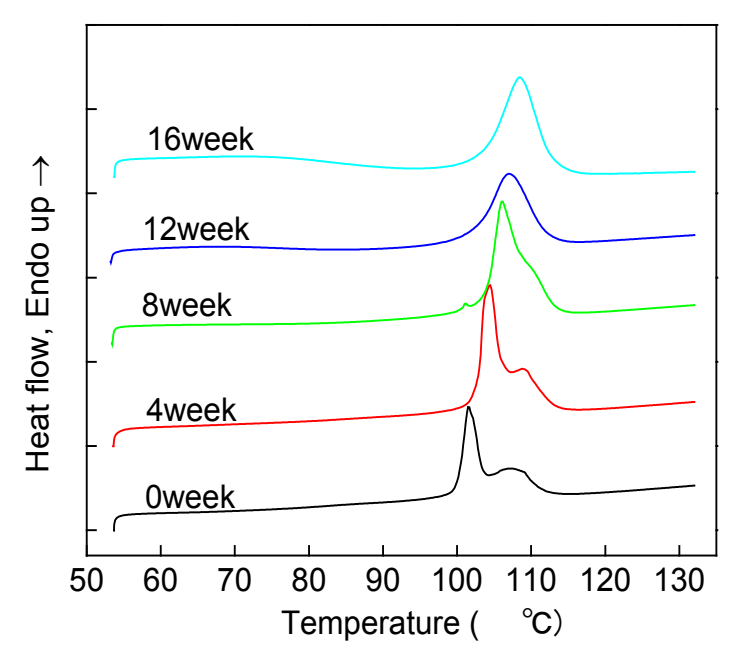

Figure 8. DSC patterns of PDO monofilament during degradation.

Table 2. $T_{M}$ and $\triangle H_{M}$ of PDO monofilament during degradation tested by DSC analysis

\begin{tabular}{|c|c|c|}
\hline $\begin{array}{c}\text { Degradation time } \\
\text { (week) }\end{array}$ & $\mathbf{T m}\left({ }^{\circ} \mathbf{C}\right)$ & $\Delta \mathbf{H m}(\mathbf{J} / \mathbf{g})$ \\
\hline 0 & $101.49 / 107.74$ & 65.61 \\
\hline 4 & $104.49 / 109.33$ & 67.58 \\
\hline 8 & $106.01 / 110.31$ & 73.20 \\
\hline 12 & 107.03 & 72.56 \\
\hline 16 & 108.54 & 71.08 \\
\hline
\end{tabular}

PDO monofilament is a property to produce wire-based stent with its favourable mechanical property and flexibility as its glass transition temperature below $0^{\circ} \mathrm{C}$. The tensile properties of PDO monofilament were shown in Figure 9. It decreased dramatically as soon as degradation started since hydrone got into molecular chains as soon as hydrolysis started to break ester bond and decrease the molecular weight. Then with the degradation going on, the morphology region was degraded and crystallinity increased, inducing continuous declination of tensile strength, only $13.44 \%$ of initial strength was left till the 10 th week. The elongation changes were in accordance to that of breaking strength and dropped $88.08 \%$ at 10 th week. Monofilament was too crispy to test at the 12 th week.

Stent mechanical properties are not only determined by monofilament tensile strength but also bending rigidity. Monofilament arranged at helix status in stent, and when come under external pressure, the bending rigidity contributed more to the stent force. Figure 10 showed the monofilament bending rigidity changes during degradation. Generally speaking, the higher the crystallinity, the higher the material rigidity. The trend of monofilament rigidity changes was consistent with the results of XRD and DSC. However, the higher the crystallinity, the monofilament was easier to be a brittle failure. At the 12th week, the sample couldn't afford the bending deformation and break off. At the 10th week, the monofilament bending rigidity increased $36.41 \%$ of initial.
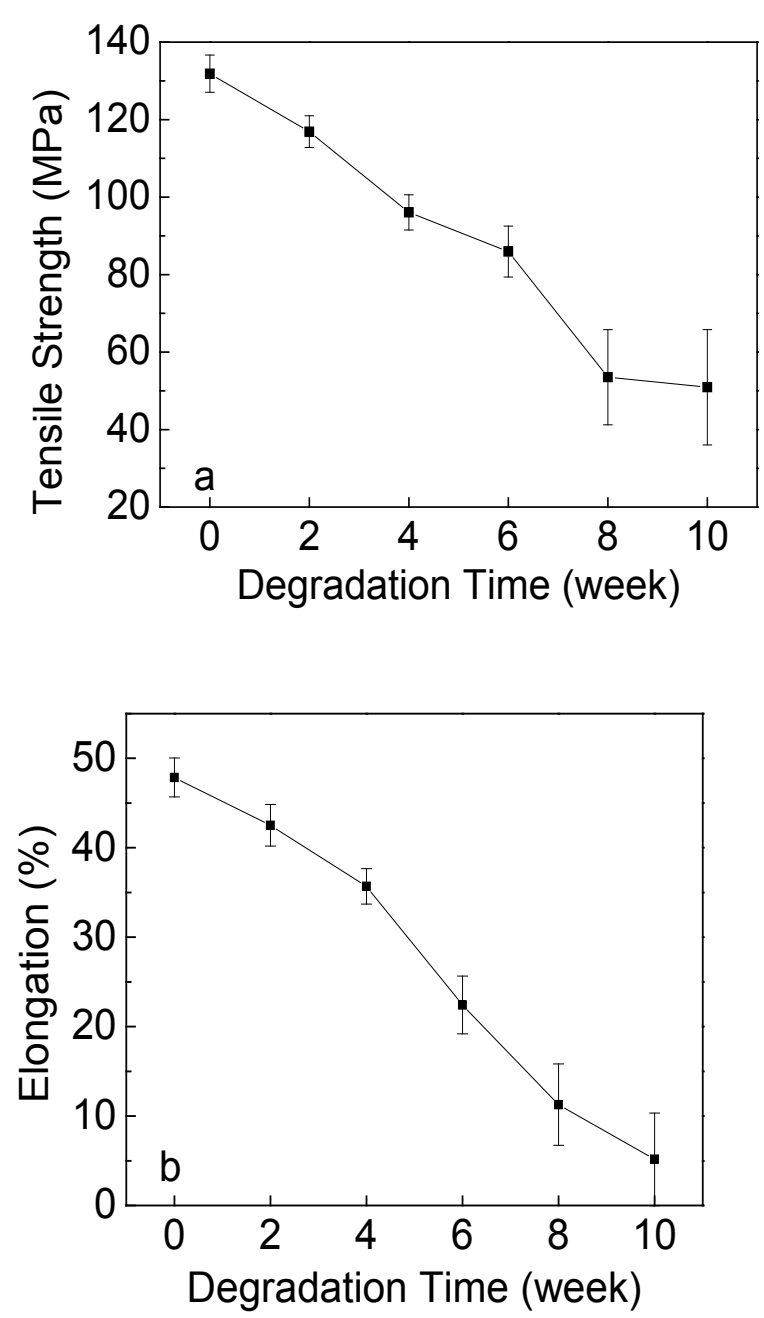

Figure 9. Tensile performance of PDO monofilament during degradation of (a) tensile strength of PDO monofilaments, (b) elongation of PDO monofilaments. Data were mean $\pm S D, n=5$.

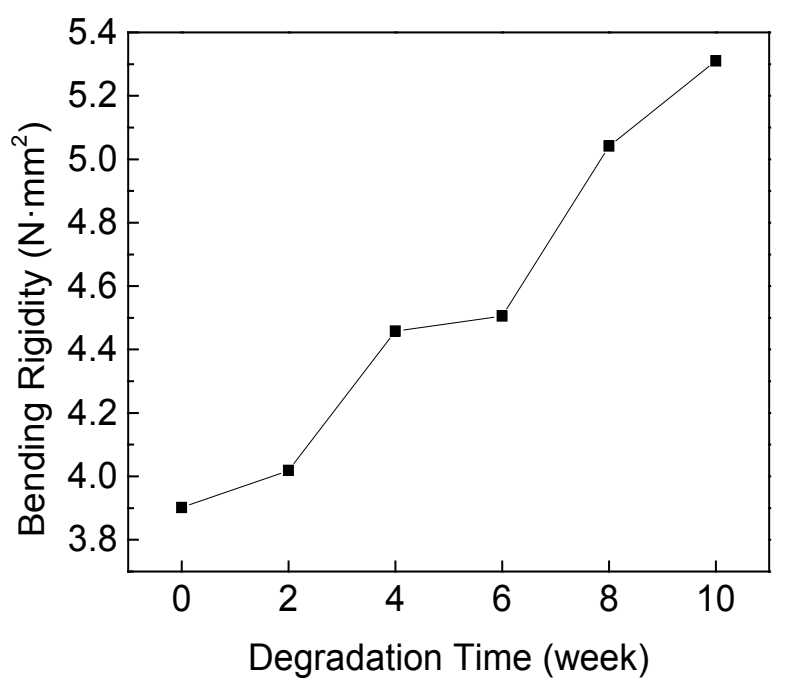

Figure 10. Bending rigidity of PDO monofilament during degradation.

\subsection{Appearance and basic properties of stent}

The appearance of stents was shown in Figure 11. It can be obviously seen from figure that the porous of stents got smaller with the increase of braiding pins. Table 3 listed the thickness, porosity and longitudinal shortage of these four groups of stents. The real tested longitudinal shortage is smaller than 
the theoretical values as the braiding angel could not be compressed to 0 . However, the change rule is consistent with the theory that stent with higher braiding pins and more compact structure get less longitudinal shortage. That's because the higher the braiding pin, there were more interlacing points to get much more friction and stable the stent structure. Also, the braiding angle $\theta$ got smaller and longitudinal deformation of rhombus unit decreased.
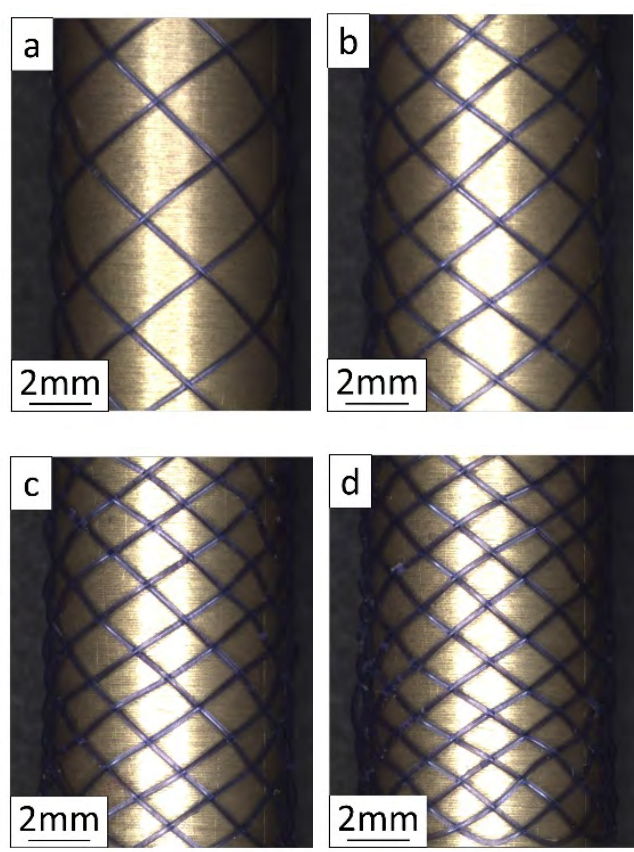

Figure 11. Appearance of PDO stents of (a) 6 pins, (b) 8 pins, (c) 10 pins, (d) 12 pins.

\subsection{Mechanical properties of stents}

After deployed in vascular, stent would bare the momentary pressure induced by human blood pressure and vascular elasticity and the long-term pressure during the healing process. So stents were expected to have sufficient radial force to support the vascular wall and not to slip or collapse. However, too much radial force would cause vascular injury. Though there is no Internet standard of stent radial force, the accepted value is higher than $30 \mathrm{kPa}$. Stents radial force in this research was shown in Figure 12. Stent $a$ and $b$ had lower force than $30 \mathrm{kPa}$, while stent $\mathrm{c}$ and $\mathrm{d}$ got the value, separately $38.46 \mathrm{kPa}$ and $35.04 \mathrm{kPa}$.

Wall Stent is a net structure stent without a fixed cross point. Monofilament was interlaced together at the status of helix by friction force instead of bonded to form the rhombus units. When stent was compressed, the radial force was supplied by monofilament bending rigidity and friction force. When the pin number increased from 6 to 8 , stent radial force significantly $(p=0.01)$ increased since stents got a more tight arrangement of monofilament and more units to bare larger $F_{1}$. However, the radial force of stent $c$ was a little higher than that of stent $d$, but not significant $(p=0.36)$, since the growth of $K$ counteracted the increase of $F_{1}$.

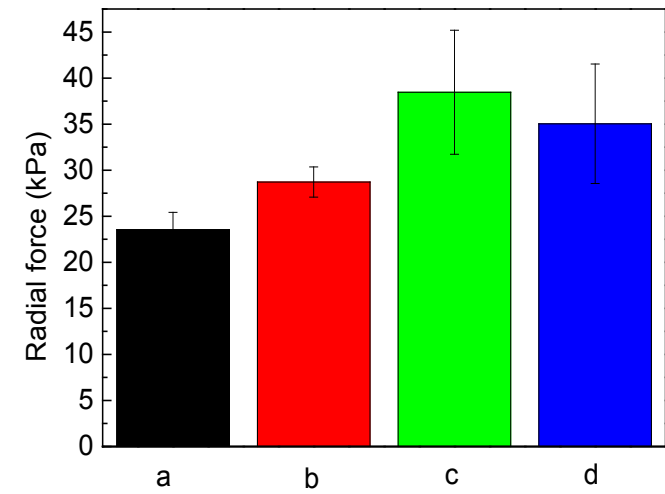

Figure 12. Radial force of PDO stents.

Figure 13 expressed the bending rigidity of stents. It can be seen that with the growth of braiding pins, stent had more compact structure to resist significantly higher $(p=0.01)$ bending force at the same deformation. Braiding pins determined the cycle number of monofilament, which would increase the resist of bending force. Stent a had lowest bending rigidity of 34.43 $\mathrm{N} \cdot \mathrm{mm}^{2}$, and stent $\mathrm{d}$ the highest of $91.35 \mathrm{~N} \cdot \mathrm{mm}^{2}$. Apart from stent $d$ had higher value, the other three groups all within the limit of $11.7-88.1 \mathrm{~N} \cdot \mathrm{mm}^{2}$.

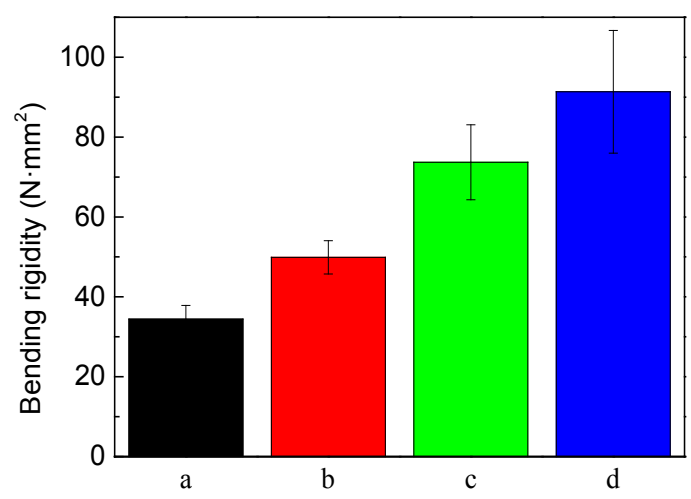

Figure 13. Bending rigidity of PDO stents.

Table 3. The basic properties of PDO stents $( \pm S D, n=5)$

\begin{tabular}{|c|c|c|c|c|}
\hline Sample & a & b & c & d \\
\hline Pin number & 6 & 8 & 10 & 12 \\
\hline Thickness (mm) & $0.59 \pm 0.003$ & $0.61 \pm 0.008$ & $0.63 \pm 0.004$ & $0.68 \pm 0.005$ \\
\hline Porosity (\%) & $87.26 \pm 0.87$ & $81.37 \pm 1.23$ & $76.76 \pm 0.69$ & $69.41 \pm 0.94$ \\
\hline Theoretical value of longitudinal shortage (\%) & 40.47 & 39.89 & 39.50 & 39.23 \\
\hline Actual value of longitudinal shortage (\%) & $38.46 \pm 1.07$ & $37.50 \pm 1.43$ & $36.91 \pm 0.96$ & $36.51 \pm 0.73$ \\
\hline
\end{tabular}




\section{$\underline{3.4 \text { Stent behaviors during degradation }}$}

Figure 14 showed the morphology change of four groups of stents during degradation process. During the first 12 weeks, there were no obvious morphology changes except lighter colored. At the 14th week, few breaking points occurred on stent $a$ and $b$, while stent $c$ and $d$ kept perfectly. At the 16th week, stent $a$ and $b$ were totally damaged, while stent $c$ were partly broken and stent $d$ still maintained integrity with few breaking points. Figure 15 showed the weight change of stents during degradation. The undegraded stents had larger weight with increase of braiding pins and significant different $(p=0.03)$. During the first 10 weeks, there were micro loss of stent weight, all below $1 \%$. Weight decreased since the 12th week and at the 14th week, small pieces of material apart from stent to cause obvious decline. At the 16th week, stent damaged and the weight decreased apparently. During the 16 weeks of degradation, stent $a$ and $b$ separately lost $17.07 \%$ and $18.06 \%$ of its initial weight, while stent c lost $15.28 \%$ and stent $d$ the lowest $13.07 \%$. Stent weight loss compared with that of monofilament (19.58\% at 16 weeks) was slower. Because the interlacing structure of stents helps resist hydrone getting in and holding small material pieces. Stent with more compact structure lost less weight as hydrone were not easy to get into the stent structure compared with sparse ones.
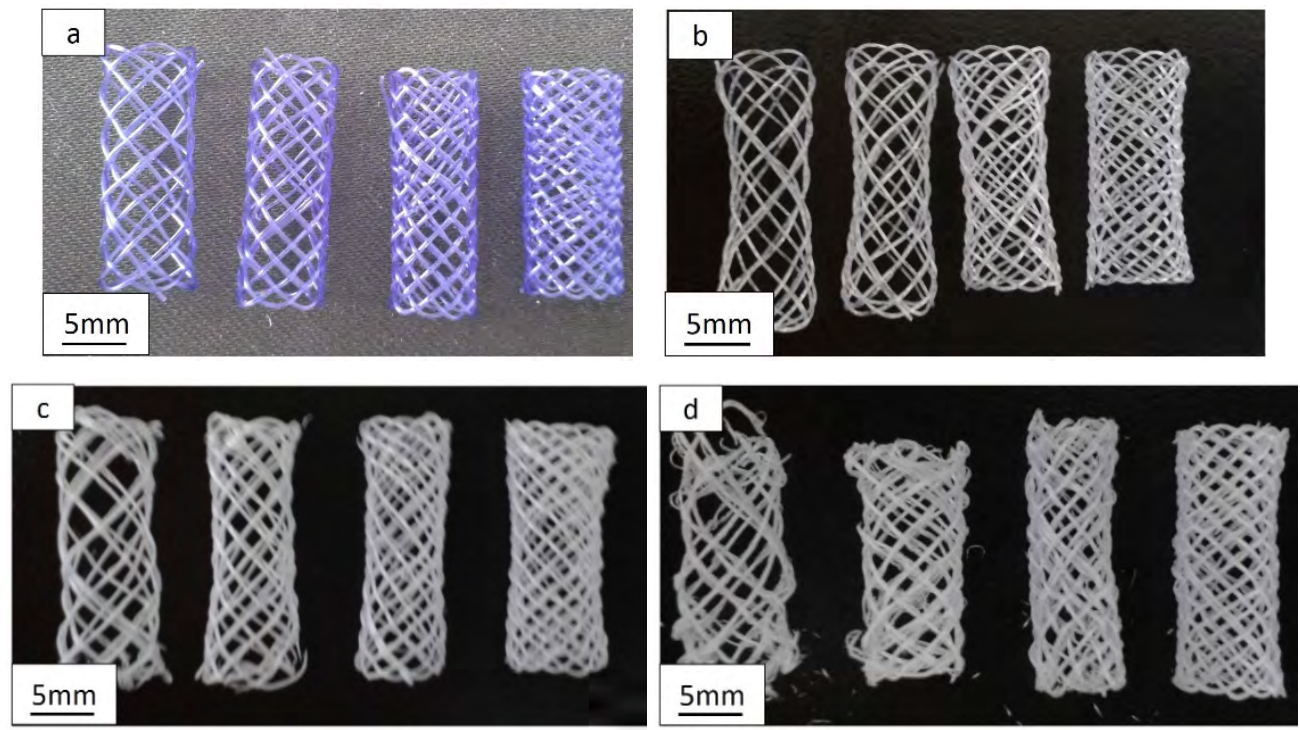

Figure 14. Appearance of PDO stents during degradation of (a) 0 week, (b)12 weeks, (c) 14 weeks, (d) 16 weeks.

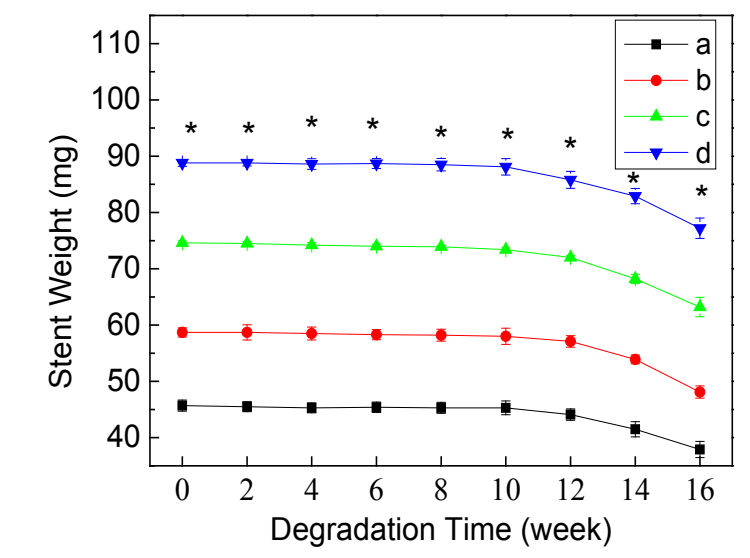

Figure 15. Weight change of PDO stents during degradation. Data were mean $\pm S D, n=5$, asterisk meant significant difference $(p<0.05, F$ test).

Stent radial force changes during degradation were shown in Figure 16. During the first 10 weeks, in addition to some fluctuations, all other three stents were on the rising trend apart from stent $a$. The radial force of stent $d$ increased apparently as the degradation started, $14.54 \%$ of growth at the second week, then fluctuated at the fourth and eighth week, increasing rapidly to $27.56 \%$ at the 10 th week. Stent b and c had similar tendency, apart from fluctuation at second and eighth week, were on a rise trend and separately increased $11.14 \%$ and $11.49 \%$ at the 10 th week. Stent a experienced slightly decline during the first 4 weeks of $8.69 \%$, then increased slowly, kept lower than original value. At the 12th week, all stents showed decrease tendency, however all other three groups were higher than initial radial force except stent a. At the 14th week, radial force of all stents was lower than its initial value. At the 16th week, apart from stent $d$, all other groups were damaged and lost much radial force, the retention of four groups was $38.62 \%$, $40.23 \%, 43.81 \%$ and $67.52 \%$, respectively, with stent $d$ the highest. During the whole period, differences between stent $c$ and $d$ were not significant, while differences among stent $a, b$ and $c$ were significant till the 16 th week.

With the process of degradation went on, the crystallinity of PDO monofilament firstly increase then decline and the turning point at the 10th week. The bending rigidity of

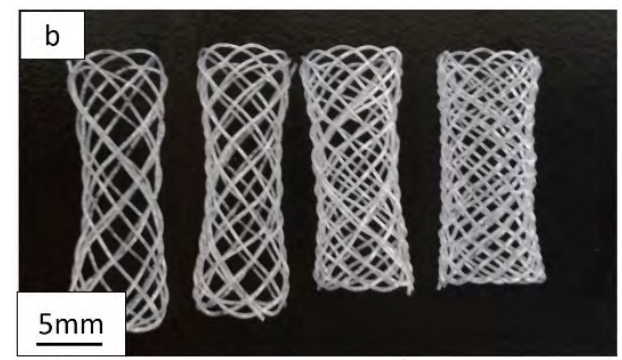


monofilament also showed increase tend till the crispy broke at the 12th week. Stent radial force experienced increase at the first 10 weeks induced by the increasing bending rigidity of monofilament. Stent with a more compact structure was more affected by monofilament bending rigidity as it is more dense helix arrangement. That explained the most obvious radial force increase of stent $d$ with the highest braiding number. After the 10th week, monofilament got weak points and easily broke, so the stent radial force decreased. At the 14th week, visible pieces broke from stent and breaking points occurred, the radial force of stents sharply decreased. With the most compact structure, some breaking points would not affect the structural integrity, so stent $d$ kept most perfectly the highest radial force at the 16 th week.

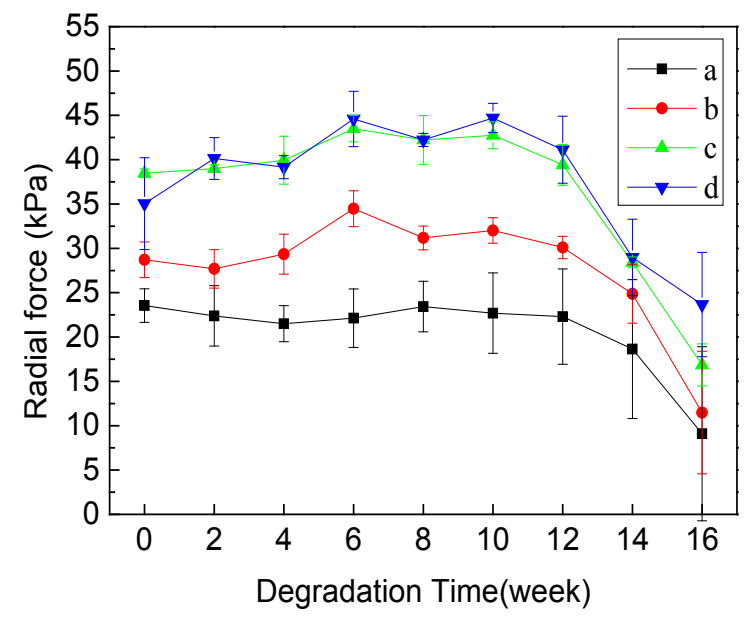

Figure 16. Radial force of PDO stents during degradation. Data were mean $\pm S D, n=5$.

Figure 17 showed the changes of stent bending rigidity during degradation. All stents showed a drop at the first four weeks with stent $a$ at the most loss of $35.34 \%$ and stent $d$ at the least loss of $14.11 \%$, while stent $b$ and $c$ lost $17.36 \%$ and $14.59 \%$ separately. During production process, PDO monofilament were wounded and twisted to cause internal stress. At the first 4 weeks, the internal stress disappeared by stress relaxation to make stents more flexible and easy to deformed at bending force. At the 6th week, stent bending rigidity started to went up except stent $c$ at the 8th week, and all stents higher than initial value except stent a $3.61 \%$ lower. Then the stent bending rigidity declined and at the 12th week, some stents occurred break points and the testing result were not significant different. At the 14th week, stent bending rigidity decreased sharply and the rigidity retention rate were separately $32.67 \%, 21.42 \%$, $40.23 \%$ and $36.51 \%$. Apart from stent d, all stents were damaged seriously during testing process. At the 16 th week, the retention of stent $d$ was $16.01 \%$ of initial value. Other three groups of stents were too broken to be tested.

The degradation behavior of stent bending rigidity was similar to that of radial force, increase firstly and then declined. Although the bending rigidity has some difference by the stress relaxation to cause the decrease of first 4 weeks. Stent mechanical behaviour was determined mainly by monofilament rigidity. During degradation, the monofilament rigidity increased with the growth of crystallinity, which would cause the increase of stent radial force and bending rigidity. With the degradation went on, the crystalline region was degraded and the monofilament subject was collapse to cause the stent damage and sharply damping of mechanical property. Stent $d$ with the most compact structure had more stable status to get highest mechanical property retention.

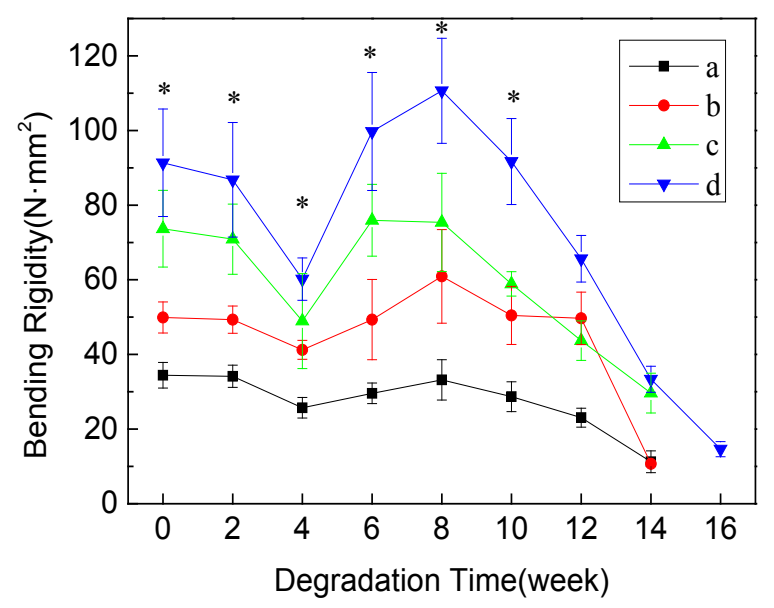

Figure 17. Bending rigidity of PDO stents during degradation. Data were mean $\pm S D, n=5$, asterisk meant significant difference $(p<0.05$, F test).

\section{Conclusion}

In the present work, biodegradable Wall Stents made from PDO monofilaments have been successfully produced with the method of mould wounding. PDO monofilaments and stents were in vitro degraded for 16 weeks. The following conclusions were established through this study:

1. Undegraded monofilament was smooth with spinning injury. Splitting and peeling occurred after 12 weeks of degradation, and obvious mass loss.

2. X-ray and DSC results showed that the crystallinity of PDO first increased during first 8 weeks and then decreased, which proved that the degradation first occurred in morphology region and then the crystalline region.

3. Monofilament tensile property failed rapidly during 10 weeks degradation while the bending rigidity increased. Monofilament tends to become hard and fragile and lost its mechanical property.

4. Stent with higher pin number had lower porosity and longitudinal shortage.

5. Stent with higher pin number had a rose and fell tendency of radial force and an increase tendency of bending rigidity.

6. Stent d maintained integrity structure after 16 weeks degradation and least mass loss.

7. Stent radial force first increased in first 10 weeks of 
degradation and then decreased, with stent $\mathrm{d}$ decreased the least.

8. Stent bending rigidity experience a fell and rose period during the first 8 weeks with the turning point at the 4th week, and then decreased till it cannot be measured, with stent $d$ decreased the least.

Therefore, it can draw a conclusion that PDO Wall Stent was able to supply effective support to vascular wall for at least 10 weeks.

\section{Funding}

This work was supported by Financial support provided by the Government of P. R. China, 111 project, "Biomedical Textile Materials and Technology" (project number B07024).

\section{References}

[1] Scot, G., Patrick, W.S. (2010). Coronary stents: current status. Journal of the American College of Cardiology, Vol. 56 (10), 1-42.

[2] Baer, G.M., Small, W., Wilson, T.S., et al. (2007). Fabrication and in vitro deployment of a laser-activated shape memory polymer vascular stent. In: Biomedical Engineering online. http://www.biomedical-engineeringonline.com/content/6/1/43

[3] Jurgen, H., Tobias, J., Hans, S., et al. (2003). Longterm outcome after implantation of bare metal stents for the treatment of coronary artery disease. Journal of Intervention Cardiology, Vol. 16 (6), 469-473.

[4] Michael, C., Jim, J., Derek, C., et al. (2006). Bare metal stent restenosis is not a benign clinical entity. American Heart Journal, Vol. 151 (6), 1260-1264.

[5] Magdalena, B.J., Bedzinski, R., Kozlowska, A. (2013). Mechanical, rheological, fatigue, and degradation behavior of PLLA, PGLA and PDGLA as materials for vascular implants. Meccanica, Vol. 48 (3), 721-731.

[6] Ormiston, J.A., Webster, M.W., Armstrong, G. (2007). First-in-human implantation of a fully bioabsorbable drug-eluting strent: the BVS poly-I-lactic acid everolimuseluting coronary stent. Catheterization and Cardiovascular Intervention, Vol. 69 (1), 128-131.

[7] Li, G., Chen, Y.F., Hu, J. (2013). A 5-fluorouracil-loaded polydioxanone weft-knitted stent for the treatment of colorectal cancer. Biomaterials, Vol. 34 (37), 9451-9461.

[8] Zamiri, P., Kuang, Y., Sharma, U., et al. (2010). The biocompability of rapidly degrading polymeric stents in porcine carotid. Biomaterials, Vol. 31 (31), 7847-7855.

[9] Marcos, A.S., Susana, G., Leni, M., et al. (2000). Study of hydrolytic degradation of polydioxanone PPDX. Polymer Degradation and Stability, Vol. 69 (2), 209-216.
[10] Chui, P.O., Ruth, E.C. (2002). The hydrolytic degradation of polydioxanone (PDS) sutures. Part l: morphological aspects. Journal of Biomedical Materials Research, Vol. 63 (3), 280-290.

[11] Stoeckel, D., Bonsignore, C., Duda, S. (2002). A survey of stent designs. Minimally Invasive Therapy \& Allied Technologies, Vol. 11 (4), 137-147.

[12] Hamm, C.W., Beythien, C., Sievert, H., et al. (1995). Multicenter evaluation of the Strecker tantalum stent for acute coronary occlusion after angioplasty. American Heart Journal. Vol. 129 (3), 423-9.

[13] Mikkonen, J., Tormala, P., Kallomaki, M. (2009). Drugeluting bioabsorbable stents-an in vitro study. Acta Biomaterialia, Vol. 5 (8), 2894-2900.

[14] Vaclav, J., Ladislav, H., Jan, H., et al. (2011). Biodegradable polydioxanone stents: a new option for therapy-resistant anastomotic strictures of the colon. European radiology, Vol. 21 (9), 1956-1961.

[15] Stivaros, S.M., Williams, L.A., Senger, C. (2010). Woven polydioxanone biodegradable stents: a new treatment option for benign and malignant oesophageal strictures. European radiology, Vol. 20 (5), 1069-1072.

[16] Prendergast, P.J., Lally, C.S., Reid, D.A., et al. (2003) Analysis of prolapse in cardiovascular stents constitutive equation for vascular tissue and finite element modelling. Journal of Biomechanical Enginerring, Vol.125 (5), 692699.

[17] Rieu, R., Barragan, P., Catherine, M., et al. (1999). Radial force of coronary stents: a comparative analysis. Catheterization and Cardiovascular Interventions, Vol. 46 (3), 380-391.

[18] Chen, M.C., Liu, C.T., Lai, W.Y. (2009). The characteristics and in vivo suppression of neointimal formation with sirolimus-eluting polymeric stents. Biomaterials, Vol. 30 (30), 79-88.

[19] Wang, Y.H., Dou, H.J., Sun, K., et al. (2010). Dynamic mechanical properties of biodegradable stents. Journal of Shanghai Jiaotong University, Vol. 44 (11), 1605-1609.

[20] Cabrera, M.S., Oomens, W.J., Bouten, V.C. (2013). Mechanical analysis of ovine and pediatric pulmonary artery for heart valve stent design. Journal of Biomechanics, Vol. 46 (12), 2075-2081.

[21] Schmidt, W., Andresen, R., Behrens, P., et al. (2004). Comparison of mechanical properties of peripheral selfexpanding nitinol and balloon-expandable stainlesssteel stents. Electronic Poster at the Annual Meeting and Postgraduate Course of the Cardiovascular and Interventional Radiological Society of Europe, Barcelona, Spain, 25-29.

[22] Ormiston, J.A., Dixon, S.R., Webster, M.W., et al. (2000). Stent Longitudinal Flexibility: A Comparison of 13 stent designs before and after balloon expansion. Catheterization and Cardiovascular Interventions, Vol. 50 (1), 120-124.

[23] Koji, M., Takashi, S. (2005). Effects of Stent Structure on Stent Flexibility Measurements. In: Annals of Biomedical Engineering, Vol. 33 (6), 733-742. 